\title{
TMTpro-18plex: The Expanded and Complete Set of TMTpro Reagents for Sample
}

\section{Multiplexing}

Jiaming Li ${ }^{1}$, Zhenying Cai ${ }^{2,3}$, Ryan D. Bomgarden ${ }^{4}$, Ian Pike ${ }^{5}$, Karsten Kuhn ${ }^{5}$, John C. Rogers ${ }^{4}$,

Thomas M. Roberts ${ }^{2,3}$, Steven P. Gygi ${ }^{1 *}$, and Joao A. Paulo ${ }^{{ }^{*}}$

${ }^{1}$ Department of Cell Biology, Harvard Medical School, Boston, MA, USA

${ }^{2}$ Department of Cancer Biology, Dana-Farber Cancer Institute, Boston, MA, USA

${ }^{3}$ Department of Biological Chemistry and Molecular Pharmacology, Harvard Medical School, Boston, MA, USA

${ }^{4}$ Thermo Fisher Scientific, Rockford, IL, USA

${ }^{5}$ Proteome Sciences, London, UK

*Corresponding author: steven_gygi@hms.harvard.edu; joao_paulo@hms.harvard.edu

Table of contents:

\begin{tabular}{|c|c|}
\hline Name & Brief caption \\
\hline Figure S1 & $\begin{array}{l}\text { MS1 ion peaks for a TMTpro-18plex reagent-labeled precursor scanned with } \\
\text { different MS1 resolutions in profile mode. }\end{array}$ \\
\hline Figure S2 & Evaluation of figures of merit in TMTpro16 and TMTpro18 experiments. \\
\hline Figure S3 & RPS6 protein abundance and gene ontology enrichment. \\
\hline Figure S4 & Raw Westernblot images. \\
\hline Table S1 & Protein quantifications in the TMTpro18 experiment. (.xlsx provided separately) \\
\hline Table S2 & Protein quantifications in the TMTpro16 experiment. (.xlsx provided separately) \\
\hline Table S3 & $\begin{array}{l}\text { Phosphorylation quantifications in TMTpro18 and TMTpro16 experiments. (.xlsx } \\
\text { provided separately) }\end{array}$ \\
\hline Table S4 & $\begin{array}{l}\text { Isotopic impurities of TMTpro-134C and TMTpro-135N. (.xlsx provided } \\
\text { separately) }\end{array}$ \\
\hline
\end{tabular}



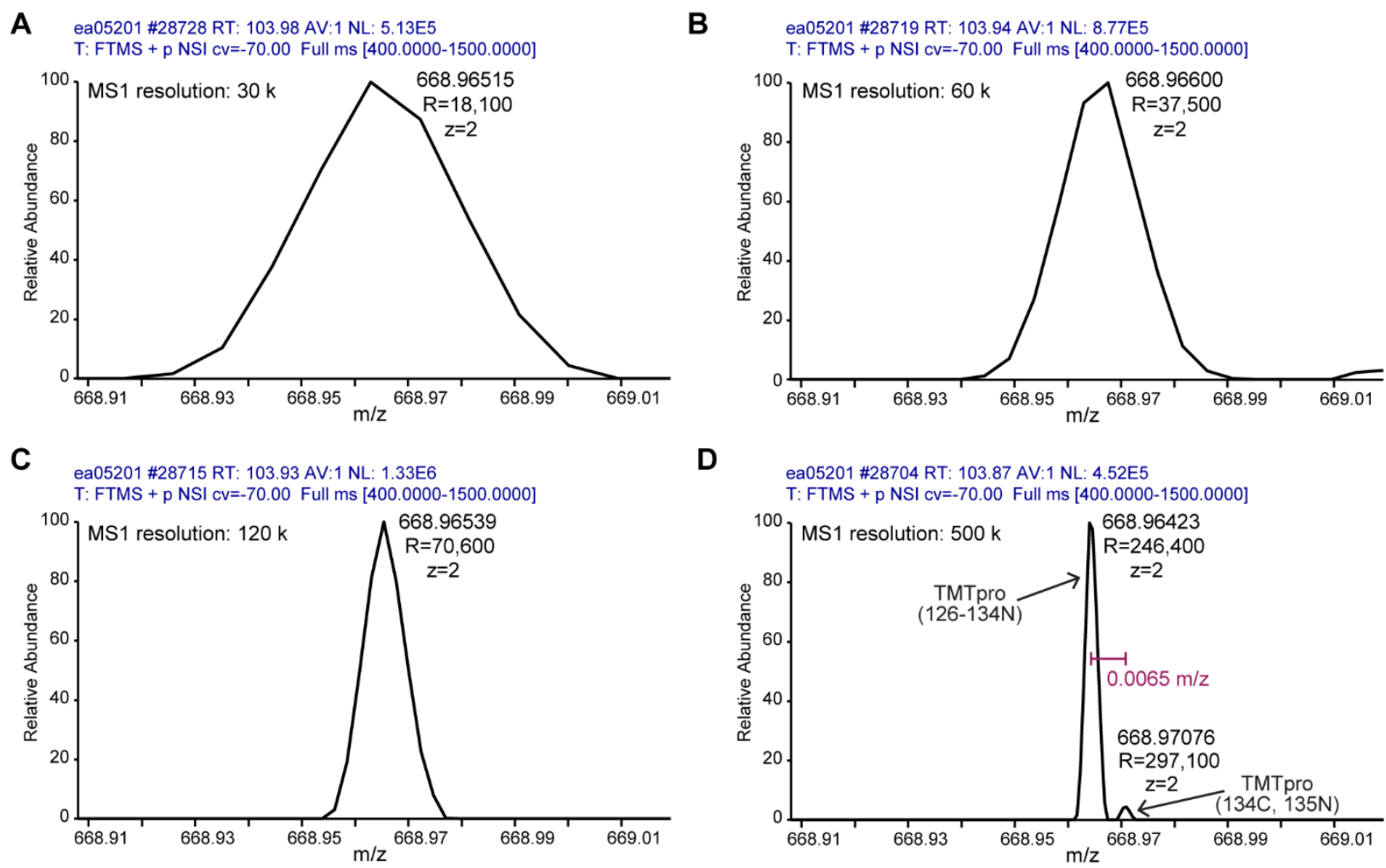

Figure S1. MS1 ion peaks for a TMTpro-18plex reagent-labeled precursor scanned with different MS1 resolutions in profile mode. The spectra originated from the 18-plex experiment in Figure 2A and consisted of 18 samples each labeled with a TMTpro-18plex reagent. The TMTpro-134C and TMTpro-135N reagent-labeled precursor (sequence: ALVILAK) has a +12 mDa monoisotopic mass shift (compared to the TMTpro16-labeled precursor) due to a difference in the number of ${ }^{15} \mathrm{~N}$ and ${ }^{13} \mathrm{C}$ isotopes (there are two TMTpro reagents attached to this peptide, so $+12 \mathrm{mDa}$ mass difference in total). The precursor is doubly charged. Commonly used MS1 resolution settings $(30,000,60,000$, and 120,000$)$ are unable to resolve the $0.006 \mathrm{~m} / \mathrm{z}$ mass difference (A-C). The MS1 resolution setting of 500,000 can detect the mass difference (D). The $0.0065 \mathrm{~m} / \mathrm{z}$ difference in (D) is the measured $\mathrm{m} / \mathrm{z}$ difference for the doubly-labeled and doublycharged peptide. The theoretical $\mathrm{m} / \mathrm{z}$ difference is $0.0063 \mathrm{~m} / \mathrm{z}$. 
A
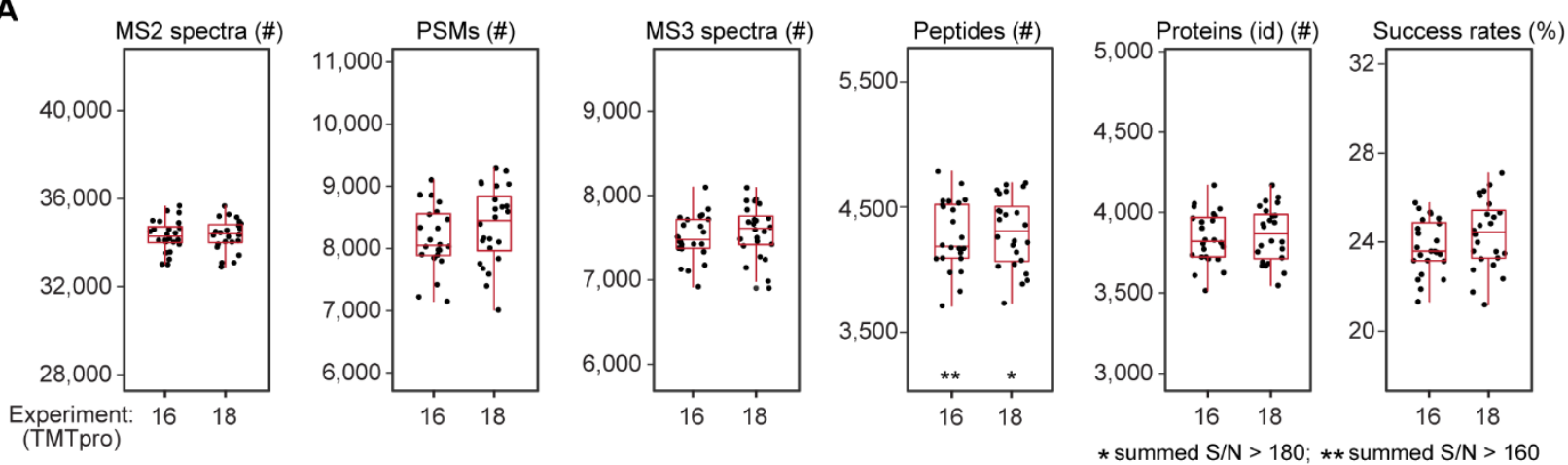

B
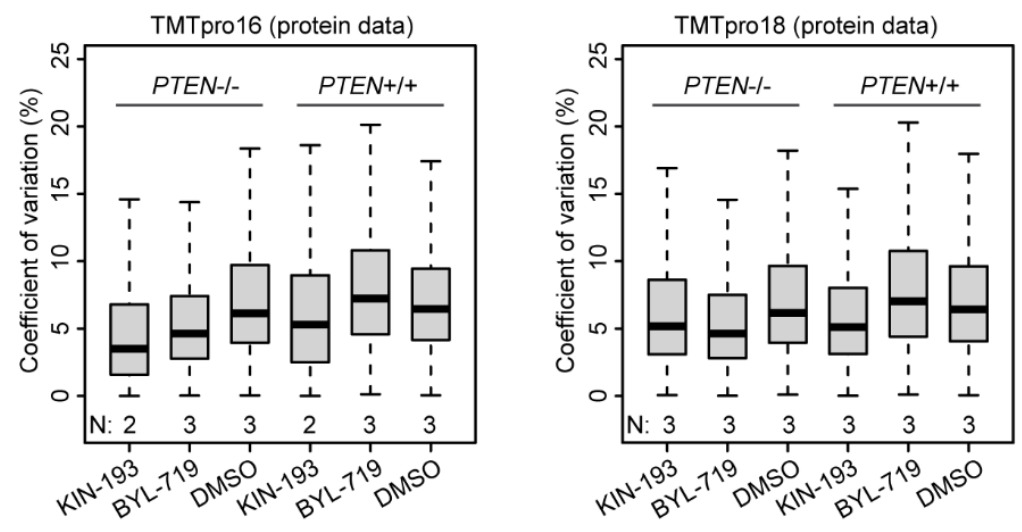

C

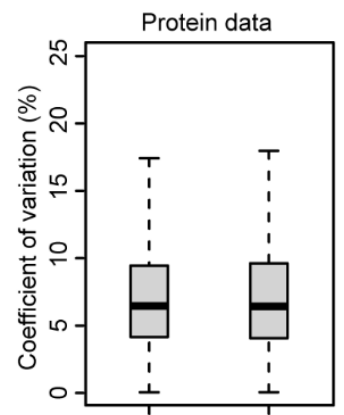

Experiment: TMTpro16 TMTpro18

Cell line: PTEN+/+ PTEN+/+

Condition: DMSO DMSO

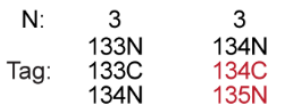

D
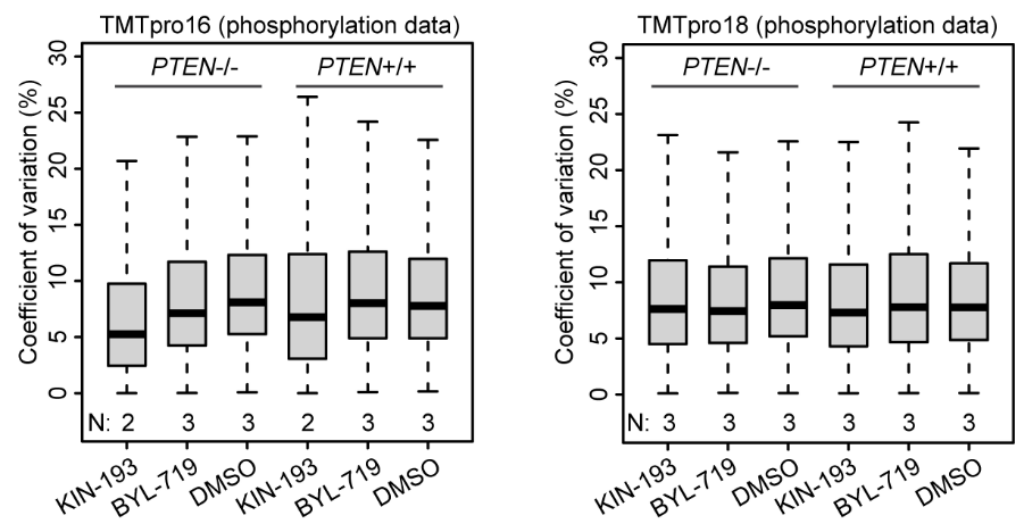

E
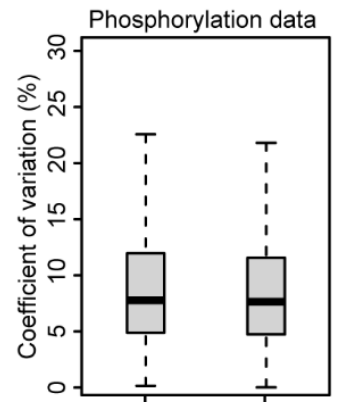

Experiment: TMTpro16 TMTpro18 Cell line: PTEN+/+ PTEN+/+ Condition: DMSO DMSO

$\begin{array}{ccc}\mathrm{N}: & 3 & 3 \\ & 133 \mathrm{~N} & 134 \mathrm{~N} \\ \text { Tag: } & 133 \mathrm{C} & 134 \mathrm{C} \\ & 134 \mathrm{~N} & 135 \mathrm{~N}\end{array}$

\section{Figure S2. Evaluation of figures of merit in TMTpro16 and TMTpro18 experiments. (A)}

The TMTpro16 and TMTpro18 experiments showed comparable numbers of MS2 spectra, peptide-spectrum matches (PSMs), MS3 spectra, peptides, identified proteins, and success rates in each fraction. Each dot represents one of twenty-four fractions. (B) Coefficient of variation 
(CV) distributions for the protein data in both experiments. A median CV of $\sim 6 \%$ was achieved in both experiments. (C) Protein CV distributions focusing on the DMSO-treated control MCF10A $(P T E N+/+)$ cell line, which include the samples labeled with the TMTpro-134C and TMTpro-135N reagents in the TMTpro18 experiment. (D) CV distributions for the phosphorylation data in both experiments. A median $\mathrm{CV}$ of $\sim 7.5 \%$ was achieved in both experiments. (E) Phosphorylation CV distributions focusing on the DMSO-treated control MCF10A (PTEN+/+) cell line, which include the samples labeled with the TMTpro-134C and TMTpro-135N reagents in the TMTpro18 experiment. 

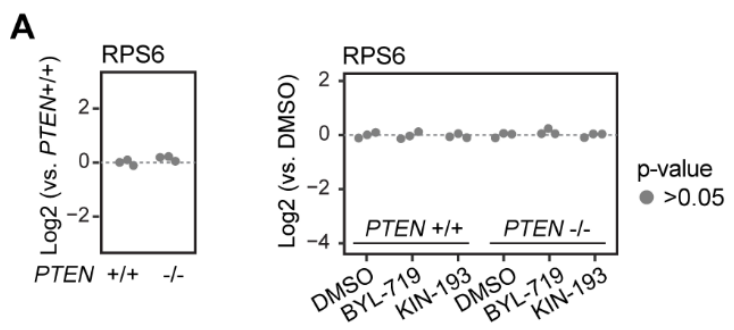

B

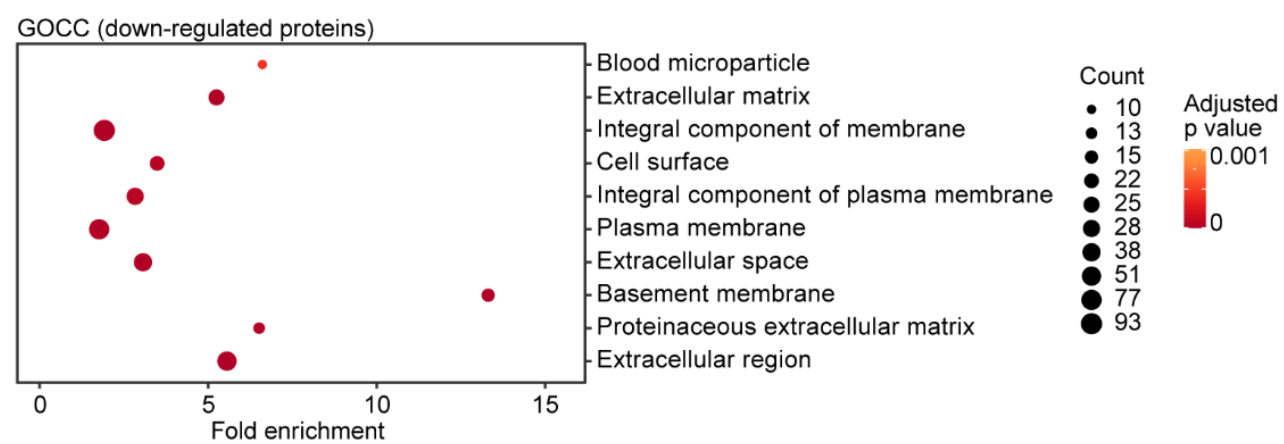

GOBP (down-regulated proteins)

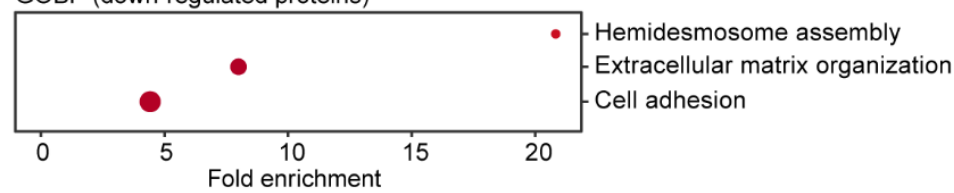

Count

- 7

71
22

GOMF (down-regulated proteins)

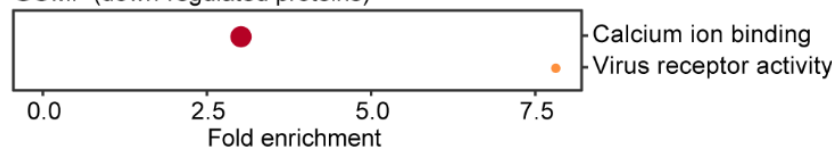

Count

- 9

GOCC (up-regulated proteins)

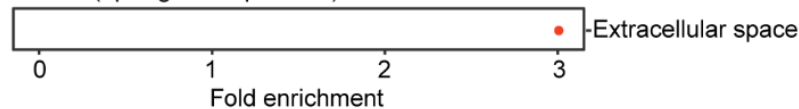

Count

Figure S3. RPS6 protein abundance and gene ontology enrichment. (A) The RPS6 protein

abundance was equivalent between control MCF10A and MCF10A PTEN-/- cell lines. The expression level of RPS6 remained unchanged after BYL-719 or KIN-193 treatments in both cell lines. (B) Gene ontology enrichment analysis of dysregulated proteins in MCF10A PTEN-/- cells (versus control MCF10A cells). 

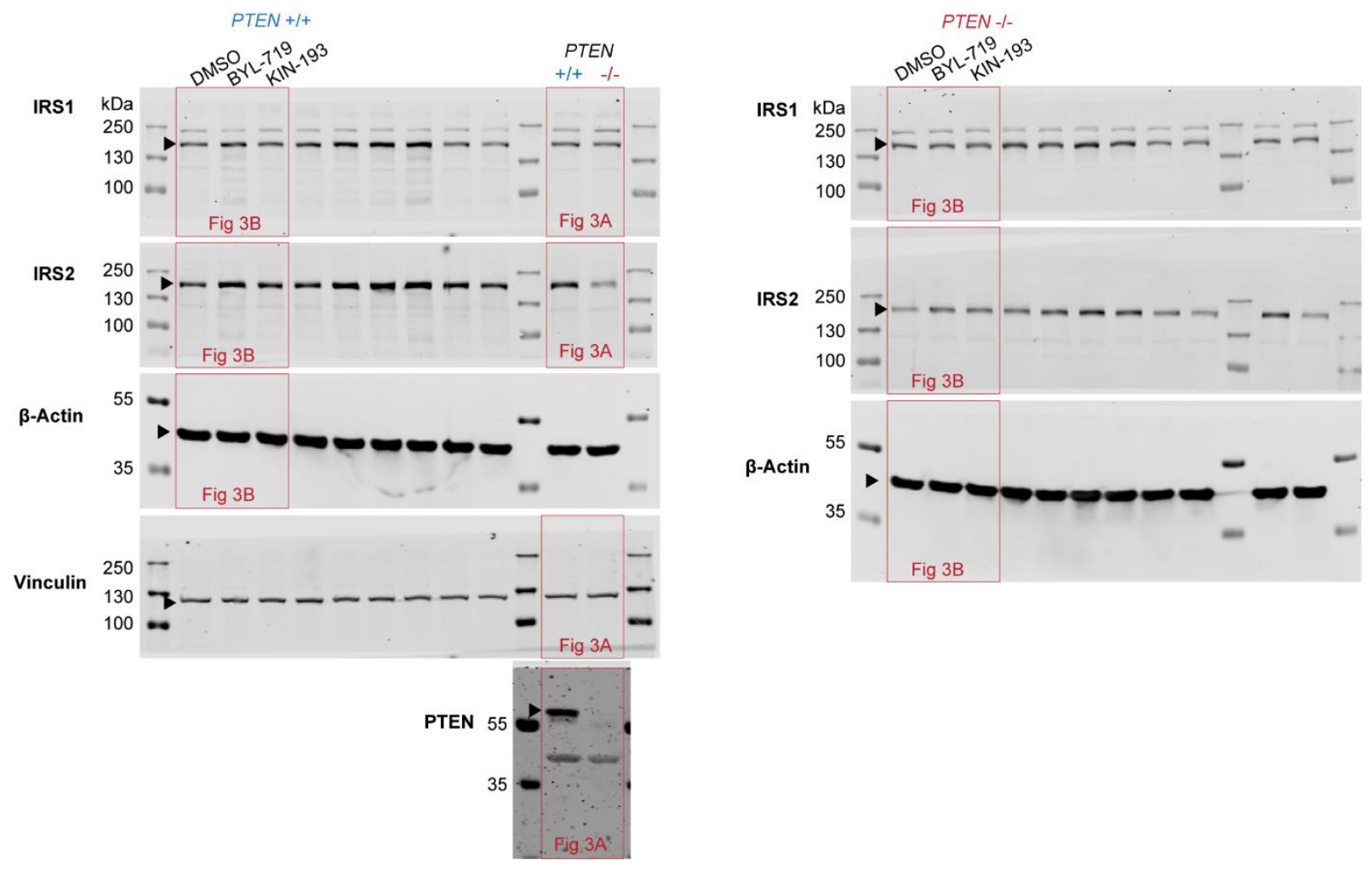

Figure S4. Raw Western blotting images. 